Meta

Journal des traducteurs

Translators' Journal

\title{
Notions essentielles et enseignement de la traduction scientifique et technique
}

\section{René Tondji-Simen}

Volume 50, numéro 4, décembre 2005

Pour une traductologie proactive - Actes

For a Proactive Translatology - Proceedings

Por una traductología proactiva - Actas

URI : https://id.erudit.org/iderudit/019905ar

DOI : https://doi.org/10.7202/019905ar

Aller au sommaire du numéro

Éditeur(s)

Les Presses de l'Université de Montréal

ISSN

0026-0452 (imprimé)

1492-1421 (numérique)

Découvrir la revue

Citer cet article

Tondji-Simen, R. (2005). Notions essentielles et enseignement de la traduction scientifique et technique. Meta, 50(4). https://doi.org/10.7202/019905ar

\section{Résumé de l'article}

Le traducteur appelé à traduire dans un domaine scientifique ou technique doit se familiariser avec les notions et la terminologie du domaine. Il doit le faire autant de fois que le nombre de domaines dans lesquels il veut traduire. Chaque fois, c'est comme s'il part de zéro. Cela lui prend énormément de temps, car les concepts sont nombreux. Les études faites en science cognitive et en terminologie ont permis de catégoriser les concepts, de dégager leurs propriétés et d'établir des critères qui permettent de rapidement analyser et comprendre les concepts. Dans le présent article, nous présentons les types et les caractéristiques des concepts ainsi que les questions fondamentales à se poser pour vite saisir les notions essentielles d'un domaine et pouvoir traduire dans ce domaine.
Ce document est protégé par la loi sur le droit d'auteur. L'utilisation des services d’Érudit (y compris la reproduction) est assujettie à sa politique d'utilisation que vous pouvez consulter en ligne.

https://apropos.erudit.org/fr/usagers/politique-dutilisation/ 


\title{
Notions essentielles et enseignement de la traduction scientifique et technique
}

\author{
RENÉ TONDJI-SIMEN \\ Collège universitaire de Saint-Boniface, Saint-Boniface, Canada \\ rtondjis@ustboniface.mb.ca
}

\begin{abstract}
RÉSUMÉ
Le traducteur appelé à traduire dans un domaine scientifique ou technique doit se familiariser avec les notions et la terminologie du domaine. Il doit le faire autant de fois que le nombre de domaines dans lesquels il veut traduire. Chaque fois, c'est comme s'il part de zéro. Cela lui prend énormément de temps, car les concepts sont nombreux. Les études faites en science cognitive et en terminologie ont permis de catégoriser les concepts, de dégager leurs propriétés et d'établir des critères qui permettent de rapidement analyser et comprendre les concepts. Dans le présent article, nous présentons les types et les caractéristiques des concepts ainsi que les questions fondamentales à se poser pour vite saisir les notions essentielles d'un domaine et pouvoir traduire dans ce domaine.
\end{abstract}

\begin{abstract}
The translator who has to do translation in a scientific or technical domain must get to know notions and terminology of the domain. He does it as many times as the number of domains in which he wants to translate. Every time, it's as if he were starting from scratch. It takes a long time as there are very many concepts. With research carried in cognitive science and in terminology studies concepts have been put into categories, their properties have been put forward and criteria which speed up the analysis and the understanding of concepts have been set. In this paper we present types and features of concepts as well as fundamental questions to ask in order to apprehend essential notions of a domain and be able to do translation in that domain.
\end{abstract}

\section{MOTS CLÉS/KEYWORDS}

concepts, questionnement, traduction scientifique et techniques

Le présent travail est appuyé de quelques exemples que nous avons utilisés dans nos cours de pratique de traduction, de traduction spécialisée et de traduction scientifique et technique. Il comprend trois grandes parties : l'acquisition des connaissances scientifiques et techniques, les notions essentielles des domaines et les questions fondamentales pour la compréhension des concepts scientifiques et techniques.

Nous partons de l'hypothèse que si vous connaissez les concepts d'un domaine de spécialité, vous comprenez les textes de ce domaine écrits dans une langue $\mathrm{D}$ et êtes en mesure de rédiger des textes du domaine dans une langue A, vous pourriez, à certaines conditions bien évidemment, faire de la bonne traduction de la langue $\mathrm{D}$ à la langue A dans ce domaine.

\section{Acquisition des connaissances scientifiques et techniques}

Sauf dans un domaine complètement nouveau, les étudiants arrivent en classe avec quelques concepts du domaine. Mais très souvent, certains de ces concepts sont mal compris, imprécis, sinon flous. Or dans les domaines scientifiques et techniques, les concepts devraient être compris de manière précise et exacte. L'apprenant devrait pouvoir placer les différents concepts dans la structure notionnelle du domaine et faire la différence entre les notions voisines ou susceptibles de porter à confusion. Par exemple, en électricité, les termes tension et intensité sont souvent confondus. De même que générateur et génératrice; travail et puissance; conductivité et conductance; isolation et isolement. Les notions auxquelles ces termes renvoient sont aussi essentielles qu'incontournables dans leur domaine, mais aussi dans beaucoup d'autres et devraient 
par conséquent faire partie de la matière du cours de traduction scientifique et technique puisque ce cours vise à former des traducteurs techniques généralistes. Les notions fondamentales et voisines telles que celles que nous venons d'énumérer, sur lesquelles les apprenants se trompent souvent doivent être abordées convenablement. Pour l'électricité, par exemple, comme le disent Reinders Duit et Christoph von Rhöneck :

l'intensité et la tension doivent être différenciées très tôt de façon à apprendre aux étudiants la notion du phénomène de flux de courant qui comporte l'idée d'un flux de quelque chose dans le circuit et celle d'une « force » directrice de ce flux, mais aussi qui permet de distinguer ces deux idées (1998: 11).

Il en est de même des notions de particule, de proton, de neutron, d'électron et du circuit électrique comme système afin de vite faire comprendre aux étudiants que lorsqu'un changement, peu en importe le type, se produit dans un point du circuit, il se produit des changements simultanés dans tous les points du circuit car les particules sont intimement interconnectées.

L'acquisition des connaissances passe très souvent par le questionnement et l'esprit de curiosité. Le dialogue (Lemke 1990 : 28) ci-après qui se déroule entre un enseignant et un de ses étudiants en est un bon exemple :

Teacher: The ground is now creating heat energy, from the light energy.

Student: How can it be the ground creates the heat energy, if the sun creates the heat energy?

Teacher: Well, on the sun, and in the sun, the sun is creating a tremendous amount of heat energy. But it's sending most of its energy here as light, traveling through space.

Student: But light is hot, light is heat.

L'étudiant ne comprend pas comment le sol peut produire de l'énergie thermique alors qu'à sa connaissance, c'est le soleil qui le fait. Mais quand l'enseignant lui répond, il se rappelle très vite que l'enseignant avait dans une discussion antérieure dit que le sol absorbe l'énergie thermique qui vient du soleil et devient chaud tout comme le sable sur une plage. Ce fait sert de prétexte pour parler de différents types d'énergie : solaire, lumineuse, biologique, biomasse, chimique, cinétique, hydraulique, hydroélectrique, électrique, éolienne, marémotrice, mécanique, photovoltaïque, résiduelle, etc.

L'enseignant revient sur sa première phrase : The ground is now creating heat energy, from the light energy pour préciser davantage les notions en jeu. Le sol ne crée pas l'énergie thermique à partir de l'énergie lumineuse. Le soleil crée l'énergie lumineuse et le sol la convertit en énergie thermique. Le sol n'est qu'un agent intermédiaire dans le processus. Nous pouvons continuer l'exercice et atteindre presque tous les domaines d'activité puisque l'énergie se retrouve partout. À mesure que nous changeons de domaine, notre connaissance de l'énergie s'approfondit et ce qui n'était pour nous qu'une notion au départ devient un ensemble de notions spécifiques. Comme le dit Lemke (1990:99):

"It is not unusual to say that teaching science is teaching the use of its conceptual systems. But just what are "conceptual systems"? Where do you go to find one? To study one? How do you build one?

Communicate one? Learn one?»

Le concept occupe une place très importante dans le processus d'apprentissage et d'acquisition des connaissances. Un concept est un élément ou une réalité perceptible d'un objet ou d'un évènement. Le concept est important car il constitue l'unité de base du sens et est, par conséquent, la composante fondamentale de la connaissance. Un objet est une substance matérielle qui peut être perçue ou utilisée par l'un ou plusieurs de nos sens. Nous pouvons le voir, l'entendre, le toucher, le goûter, le sentir. Un événement est, quant à lui, ce qui arrive à l'objet. Les événements et les objets sont liés, car les événements ont toujours besoin d'objets 
pour se réaliser. L'un des postulats de base en science naturelle est le principe de cause à effet qui signifie que les choses ne se produisent pas d'elles-mêmes, que la nature comprend la substance et l'énergie et que tous les événements et toutes les choses résultent de l'interaction entre la substance et l'énergie (Mintzes 2000 : 50). Encore l'énergie! Que pourrions-nous faire sans elle? Voilà, il faut le dire, un concept essentiel presque à tout.

Le développement de la structure cognitive procède habituellement de haut en bas et de bas en haut. Un apprenant saisit d'abord les concepts un peu généraux (par exemple, les chiens). Ensuite, il passe aux concepts plus précis ou spécifiques (par exemple, les colleys), puis à ceux qui sont plus larges (par exemple, les canidés ou les mammifères). Un bon apprentissage de concepts superordonnés permet de voir et de comprendre les concepts qui lui sont subordonnés. L'acquisition des concepts superordonnés fondamentaux doit être l'objectif principal de l'enseignement des sciences (Mintzes $2000: 6$ ).

\section{Compréhension du concept en science}

La pensée humaine comprend implicitement la connaissance et l'aptitude à utiliser cette connaissance pour réagir aux événements, en prédire les résultats et prévoir les choses. La connaissance est un ensemble de faits, de principes, de significations, de concepts et de relations qu'un individu possède en vue d'une activité déterminée (TERMIUM). Les éléments de la connaissance sont représentés dans la langue par des symboles et permettent d'exprimer ou de comprendre le monde. La représentation et le traitement sémantiques sont nécessaires pour comprendre ou produire des messages linguistiques, $d$ 'autres actions et d'autres expressions symboliques, acquérir, extraire ou utiliser les connaissances conceptuelles, représenter, planifier et contrôler les procédures et les actions afin d'atteindre des objectifs spécifiques. En d'autres termes, pour comprendre une expérience, il faut accéder au réseau conceptuel et au schéma de cette expérience. Le schéma se définit comme une grappe de connaissances qui comprend un ensemble de concepts et les relations qui les unissent et qui appartiennent tous à un concept plus complexe. L'apprentissage consiste à mettre en rapport les nouvelles expériences et celles qu'on connaît déjà. La personne qui comprend un concept est celle qui en a une vue d'ensemble et non une vision parcellaire ou fragmentaire. Les apprenants qui réussissent mieux sont ceux qui arrivent à percevoir ou à établir des relations hiérarchiques systématiques entre les objets et les événements considérés, à bien utiliser les relations spécifiques, regrouper et classer les informations par type, et à établir la différence entre des éléments apparentés ou proches. Les apprenants doivent comprendre les concepts d'importance, savoir structurer, organiser, conserver, extraire et utiliser l'information.

\section{Nature des relations}

Trois relations suffisent pour caractériser la plupart des concepts scientifiques fondamentaux : les relations ontologiques (relations partie/tout) fondées sur la contiguiité ou le contact dans l'espace et dans le temps, les relations logiques (relations ensemble/élément) fondées sur la ressemblance et les traits distinctifs. Chaque domaine ou sous-domaine a ses concepts et ses expressions. Il n'est pas facile de maittriser les relations qui relient les concepts. Cette tâche est encore plus difficile pour les traducteurs qui, pour la plupart, n'ont pas assez de connaissances dans le domaine et pour qui la langue du texte qu'ils vont traduire est une deuxième ou une troisième « langue seconde ». Or, comme nous le savons, lorsqu'on apprend une seconde langue, on apprend les noms avant les verbes. Les concepts sont généralement représentés par les noms et les relations sont exprimées par les verbes (Mintzes 2000 : 204). C'est quand l'apprenant a compris le nom qu'il est en mesure de comprendre les relations et les phraséologismes auxquels le nom est partie prenante. Les cours de traduction spécialisée doivent beaucoup insister sur l'apprentissage des concepts. 


\section{A) Les relations logiques}

Les relations logiques sont les relations qui existent entre deux concepts qui se ressemblent et qui partagent certains de leurs caractères. Ces relations sont de deux types :

a) La relation générique/spécifique : le générique a un sens plus large et le spécifique prend tous ses caractères et y ajoutent des caractères qui le singularisent. Cette relation est aussi appelée relation hyperonyme/hyponyme. Exemple : énergie - énergie thermique. C'est une relation verticale ou hiérarchique.

b) La relation horizontale : elle caractérise les notions qui ont un même générique. Ces notions ont chacune, tous les caractères du générique mais chacune d'elles a des caractères qui la distinguent de l'autre. Cette relation est aussi appelée co-hyponymie. Exemple : énergie thermique - énergie nucléaire.

\section{B) Les relations ontologiques}

Les relations ontologiques reposent sur la proximité spatiale des éléments. Elles sont de deux types :

a) Les relations partitives qui comportent deux types de relations : celles qui existent entre un tout et ses parties. Exemple : Moteur : arbre, bielle, carburateur, culasse, distributeur, piston. Et celles qui existent entre les différentes parties d'un même tout (arbre, bielle, carburateur, culasse, distributeur). Ces relations sont basées sur la proximité spatiale.

b) Les relations d'enchaînement, qui reposent sur une succession d'événements dans le temps (relations de cause à effet), et qui sont, par conséquent, séquentielles (Cabré, 1998 : 179).

Les relations ontologiques peuvent être divisées en de nombreux sous-types plus précis dont certains sont présentés dans Cabré (1998 : 180) et d'autres dans L'Homme (2004 : 100102) : composant - objet (brique - mur), élément - ensemble, membre - collection (joueur - équipe), partie fonctionnelle - tout, portion - masse (portion - pizza), matière - objet (coton - vêtement), lieu - région (Valcartier - Québec), lieu - zone (cerveau - boîte crânienne), phase - processus (adolescence - maturation), contenant - contenu (bouteille - vin), constituant - objet (porcelainecaoutchouc - pneu), matériau - produit (acier - poutre), matériau - propriété (verre - fragilité), matériau - état (fer - corrosion), procédé - produit (tissage - tissu), procédé - instrument (incision - scalpel), procédé - méthode (entreposage - séchage à froid), procédé - patient (teinture - textile), phénomène - mesure (lumière - watt), objet - contreagent (poison - antidote), objet - qualité (pétrole - indice d'octane élevé), objet - opération (vrille - vrillage), objet - caractéristique (fuel - sans fumée), objet - forme (livre - couverture souple).

Il existe aussi une autre typologie fine des relations comprenant les relations physiques (sous, près, etc.), relations temporelles (précède, suit) (Saadani et Bertrand-Gastaldy 2000 : 13). Les relations non-hiérarchiques sont très importantes dans la représentation des connaissances dynamiques :

Exemples :

\begin{tabular}{|l|l|}
\hline Relation & Example \\
\hline Cause - effect & Smoking - malignant tumor \\
\hline Activity - place & Treatment - hospital \\
\hline
\end{tabular}




\begin{tabular}{|l|l|}
\hline Relation & Example \\
\hline Object - form & Cancer cell - oat cell \\
\hline Process - method & Diagnostic test - bronchoscopy \\
\hline Method - instrument & Bronchoscopy - bronchoscope \\
\hline
\end{tabular}

FABER, Pamela (1999 : 13)

\section{C) Autres présentations des relations}

- On peut distinguer les choses par la couleur (RED APPLE/YELLOW APPLE), par l'âge (OLD APPLE/NEW APPLE), par le type ou la classe (MACINTOSH APPLE/APPLE), etc. - On peut diviser les relations sémantiques en cinq groupes :

- les attributs (qualité, quantité, type; exemple : the three red Macintosh apples);

- les relations taxonomiques : synonymie (équivalence), antonymie (opposition), hyponymie (générique/spécifique, partie/tout);

- les relations entre les processus, les activités et les objets ou les agents qui y participent, le but, le moyen, la finalité, la portée, l'étendue, l'identification (A est B) et la possession (A a B);

- les relations circonstancielles : lieu, temps, manière, motivation, matière, moyen, etc.;

- les relations qui indiquent les rapport : cause/effet, preuve/conclusion (évidence/conclusion), général/particulier, abstrait/concret, etc.

- La relation [est une parties de] est la Méronymie, [est un type de] est la Classification, [par exemple] est l'aspect/le type, [au nombre de] est la Quantification, [dans, à, sur, sous] est le Lieu, et [a, possède] est la Possession (mais peut aussi indiquer la méronymie).

\section{Types de concepts}

Selon la typologie de Frank C. Keil (1989 : 25), il y a des concepts de types naturels (classes d'objets naturels qui existent indépendamment des activités humaines. Exemples : animaux, plantes mais aussi des éléments et des composés), des concepts de types nominaux ou conventionnels (concepts dont le sens dépend grandement des conventions sociales ou des stipulations) et des concepts d'objets fabriqués ou artificiels tels que les meubles, les vêtements, les véhicules. Il ajoute à propos des concepts nominaux qu'il s'agit des idées abstraites que nous avons dans notre esprit, c'est-à-dire des concepts que nous pouvons avoir par exemple sur le cheval, le plomb, et les modifier sans que le cheval et le plomb ne changent d'espèce (Keil, 1989 : 37).

La norme ISO 2788 1986, quant à elle parle : 1) des entités concrètes (les objets et leurs parties physiques et les matériaux), 2) des entités abstraites (les actions et les événements; les propriétés des objets, des matériaux ou des actions; les disciplines ou sciences; les unités de mesure) et 3) des entités individuelles ou «classes d'un seul individu» comme les noms propres. À cette liste, Saadani et Bertrand-Gastaldy (2000 : 8).ajoutent les processus, les sentiments, les idées, les constructions, les relations, les qualités ou les propriétés, les quantités, les procédures et les produits.

Howard classe les concepts en deux grandes catégories : les objets et les événements. Il définit les objets comme des choses physiques qui se trouvent en un lieu imaginaire ou réel. Exemples : planète, microbe, virus, voiture (Howard 1987 : 23). Les événements se situent dans le temps et comportent un ensemble ou une succession d'actions. Exemples : manger, courir. Les concepts peuvent être concrets ou abstraits. Les concepts concrets sont très liés à nos sens et sont 
donc perceptibles : rouge, table, chien. Les concepts abstraits sont un peu difficiles à cerner et leur contenu notionnel peut varier d'une personne à une autre. Exemples : justice, liberté, beauté, démocratie. Les concepts peuvent aussi être classés selon les domaines auxquels ils appartiennent. Exemples :

- concepts scientifiques : ion, gravité, molécule.

-.concepts mathématiques : racine carré, chiffre impair, cosinus, sinus.

- concepts juridiques : égalité devant la loi, droit naturel, droits successoraux.

\section{Analyse du concept}

Les caractères du concept correspondent, comme le précise la norme ISO/R 1087, à chacune des propriétés qui le décrivent.

Il s'ensuit que les concepts se différencient entre eux parce qu'ils possèdent des caractères différents. Une description conceptuelle satisfaisante doit tenir compte des traits qui permettent de distinguer entre concepts du même niveau. (Cabré 1998 : 169).

Exemple :

énergie mécanique : énergie due au mouvement de corps, dont on peut trouver des exemples dans le déplacement d'un courant d'eau ou la rotation d'un solvant d'inertie (TERMIUM).

énergie hydraulique : énergie cinétique, d'origine potentielle, contenue dans un écoulement d'eau (TERMIUM).

énergie hydroélectrique : énergie électrique qui provient de la transformation de l'énergie hydraulique (TERMIUM).

énergie éolienne : énergie mettant en œuvre le mouvement des masses d'air dans l'atmosphère (Grand Dictionnaire terminologique).

« La présence (ou l'absence) d'un caractère à la place d'un autre dans un ensemble de caractères reliés entre eux rend compte de la différence entre concepts d'un même système conceptuel, comme nous l'observons dans les cas cités ci-dessus » (Cabré $1998: 169)$.

\section{Description du concept}

On peut décrire un concept par compréhension ou par extension : « La description d'un concept par compréhension [...] consiste à énumérer tous les caractères qui le décrivent du plus générique au plus spécifique. Ainsi, par exemple, la compréhension du concept » (Cabré 1998 : 173-174) «chien» correspond à l'ensemble formé par les caractères suivants : chien : mammifère - carnivore - canidé

« La description d'un concept par extension consiste à énumérer toutes ses réalisations possibles » (Cabré 1998 : 174). Exemple : Dent : canine, incisive, molaire

\section{Types de caractères}

Le concept se compose d'une série de caractères qui sont communs à une classe d'objets individuels. Ces caractères nous servent à structurer la pensée et à la communiquer. Les concepts se regroupent dans des ensembles (les champs conceptuels) qui partagent plusieurs caractéristiques. (Cabré, 1998 : 84)

a) «Selon la pertinence qu'ils manifestent dans la constitution d'un concept, les caractères peuvent être essentiels ou complémentaires. Les caractères essentiels d'un concept sont ceux qui décrivent son essence, et qui sont indispensables pour la décrire » (CABRÉ, 1998 : 170). Les caractères non essentiels ou complémentaires d'un concept comportent des éléments non pertinents à la description. 
b) «Selon la relation qu'ils entretiennent avec l'objet qu'ils représentent, les caractères des concepts peuvent être intrinsèques ou extrinsèques.

Les caractères intrinsèques d'un concept sont ceux qui sont liés à sa description comme représentant une classe : forme, couleur ou moyen, dans le cas des noms d'objets; type d'action, de sentiment ou de processus, dans le cas des verbes, etc.

Les caractères extrinsèques d'un concept, par rapport aux intrinsèques, sont externes à sa définition comme classe : fonction, origine, destination, localisation, inventeur, etc. »

c) «Les caractères peuvent également être dépendants ou indépendants. Les caractères dépendants doivent nécessairement intervenir à des niveaux différents de la hiérarchie, ou, comme le souligne Felber (1987 : 100), le caractère supérieur doit précéder le caractère subordonné. En revanche, les caractères indépendants peuvent se situer à différents niveaux d'une série verticale et être combinés arbitrairement (Felber 1987 : 101) » (CABRÉ, 1998 : 172).

Les concepts ont plusieurs types d'attributs dont trois sont très importants : les attributs physiques (attributs perceptibles par un ou plusieurs de nos sens : les attributs d'un arbre, ce sont les racines, un tronc haut et des branches), les attributs de fonctions (attributs révélant à quel usage le concept est destiné : l'attribut d'un vélo est qu'il est fait pour transporter quelqu'un d'un point à un autre) et les attributs relationnels (attributs qui indiquent l'existence d'une relation entre deux concepts : 1'attribut de voisin c'est le lien de proximité entre deux ou plusieurs personnes ou choses).

Les attributs peuvent aussi être classés en trois catégories : les attributs définitoires (attributs qu'un objet doit absolument avoir pour être un exemple d'un concept : le globule rouge est un attribut définitoire du sang), les attributs caractéristiques (attributs que la plupart d'instances d'un concept ont mais pas toutes : voler caractérise les oiseaux mais tous les oiseaux ne volent pas) et les attributs non pertinents (attributs qui ne déterminent pas du tout l'appartenance d'une instance à un concept : la couleur n'est pas pertinente pour distinguer un numériseur d'une imprimante (Howard 1987 : 21)

\section{Notions essentielles des domaines}

Les connaissances du monde se ramènent à des concepts. Qui accède aux concepts accède aux connaissances. Le traducteur (en formation ou en milieu professionnel) se trouve confronté aux difficultés voire à l'opacité des textes scientifiques et techniques. Très souvent, il doit parer au plus pressé. Les mots sont comptés, le temps aussi. Il s'attaque de front aux textes. Il peut s'être constitué un « trésor de guerre »: vocabulaires, glossaires, lexiques, banques terminologiques, encyclopédies et autres nombreux ouvrages. Il peut très bien tirer profit de ce trésor mais au prix de beaucoup d'efforts, de recherches, de lectures et de nombreux sacrifices. Il a déjà expérimenté plusieurs méthodes de formation ou de travail. Il lui faut en expérimenter une autre, celle qui tient compte de ce qui a été dit dans la première partie du présent travail.

Les concepts structurent les domaines de connaissance. On peut les sonder, les analyser, les décrire et les interpréter. Pour le faire, il faut recourir aux caractères intrinsèques et extrinsèques, et aux différentes relations sémantiques analysées plus haut. Certaines relations et certains caractères conviennent mieux aux objets et d'autres aux événements. La relation partitive, par exemple, s'applique bien aux systèmes et organes du corps, aux lieux géographiques, aux disciplines ou aux domaines des connaissances et aux structures sociales hiérarchisées ( Saadani et Bertrand-Gastaldy 2000 : 10). La forme, la couleur, la taille, le poids, la matière, le matériau, le genre, l'espèce, la composition, etc. constituent les caractères intrinsèques d'objet. La fonction, l'origine géographique, la destination, la localisation, l'inventeur, l'utilisateur, l'utilisation, la finalité, etc. déterminent les caractères extrinsèques d'objet. Et le processus, le début, la fin, l'action, la cause, l'effet, etc. permettent de caractériser les événements. Chaque domaine a sa structure (générique/spécifique, partie/tout), ses objets (instruments, outils), ses événements (activités). 
Chaque science a des questions de base auxquelles elle répond. Il faut pouvoir en identifier. Par exemple : la structure de base de la matière, la masse et la force, l'énergie, la troposphère, l'électricité en physique; le règne animal, les gènes, les chromosomes, le mécanisme de l'évolution et la vie des cellules en biologie; la structure de l'univers et la vie des étoiles en astronomie; les nombres réels, les nombres imaginaires, les, limites, la corrélation en mathématique; l'ion, la base, la solution en chimie; l'algorithme, le langage de programmation, la structure des bases de données en informatique. Les étudiants aiment apprendre les concepts qui existent dans un domaine, savoir comment ces concepts se relient les uns aux autres et quelle est leur taxonomie.

Dans chaque taxonomie, il y a des concepts fondamentaux. Ces concepts sont habituellement les plus usuels dans le domaine et c'est eux qu'on apprend en premier. Les concepts au-dessus (les superordonnés) sont plutôt abstraits et ceux en dessous (les subordonnés?) spécifiques. Plus on descend dans la taxonomie, plus on obtient de l'information par concept. Plus on monte, plus les concepts deviennent abstraits et moins on obtient de l'information.

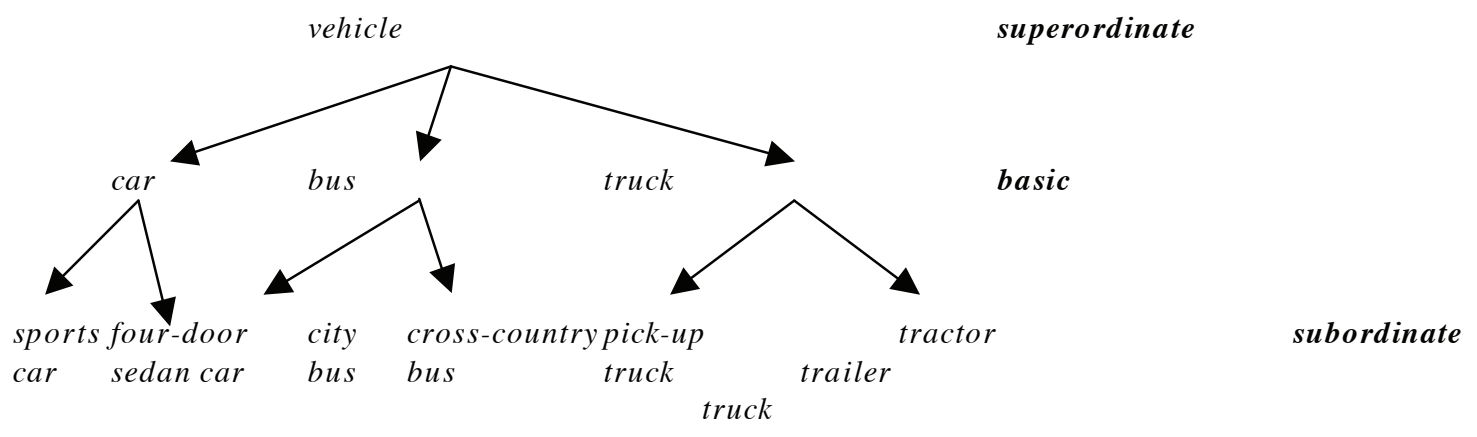

Taxonomie de véhicule montrant le superordonné, les concepts fondamentaux et les subordonnés (Howard 1987 : 54).

L'enseignement des concepts se fait mieux si l'on commence par les concepts fondamentaux. Ils sont plus faciles à assimiler. Ensuite, on peut aller vers le haut ou vers le bas selon l'objectif qu'on veut atteindre. Sans oublier qu'on peut aussi aller à l'horizontale pour les cohyponymes, ou les concepts qui se situent au même niveau d'abstraction mais qui sont différents les uns des autres comme sports car et four-door sedan car dans la taxonomie cidessus ou melting, boiling, freezing, dissolving et condensing dans la taxonomie de changement d'état en physique ou en chimie minérale. Dans une procédure classique, le professeur détermine d'abord les caractéristiques du concept à enseigner et les enseigne aux étudiants. Il présente ce concept dans les relations qu'il a avec les autres concepts du domaine. Ainsi, s'il faut parler des espèces animales, il faut bien montrer que dans la taxonomie les espèces se placent en dessous du genre et de la famille et au-dessus des sous-espèces.

Quand vous abordez un domaine ou un thème, il y a toujours un concept de départ. Ce concept peut être un objet ou un événement. Quel que soit le domaine que vous abordez, prenez ce concept de départ (le premier concept auquel vous accédez) et posez-vous des questions à son sujet. De quoi s'agit-il au fond? Est-ce un objet, un événement? Si c'est un objet, est-ce un générique? Si c'est un générique, quels en sont les spécifiques? Quels sont ses caractères? Qu'est-ce qui distingue les spécifiques entre eux ou par rapport au générique. Si l'objet en question est un tout, quelles en sont les parties et quels sont leurs rôles respectifs? Avons-nous à faire à un ensemble d'éléments qui n'entretiennent que des relations situationnelles de proximité? Et pourquoi cette proximité? 


\section{Questions fondamentales pour la compréhension des concepts scientifiques}

Comme nous venons de le voir, nombreuses sont les questions qu' on peut se poser pour une bonne compréhension des sciences et des techniques. Mais toutes ces interrogations peuvent se ramener aux six questions fondamentales que nous entendons beaucoup dans la vie courante mais que nous ne nous posons pas quand il est question des sciences et des techniques. Nous serions pourtant surpris de l'apprentissage que nous ferions en répondant de manière précise et spécifique aux six questions suivantes : QUI, QUOI, OÙ, QUAND, COMMENT, POURQUOI. Ces questions d'investigation ont une puissance presque « chirurgicale » pour découper le réel et dégager les faits (Bellenger 2000 : 34). Pour l'écrivain anglais et prix Nobel Rudyard Kipling ces questions sont « les six fidèles serviteurs qui dirigent toute sa vie ». Pour trouver des réponses, il faut savoir poser les bonnes questions.

Deux types de questions sont au cœur de la recherche d'information scientifique : 1) le QUOI; 2) le COMMENT et le POURQUOI.

1) La question QUOI se pose au niveau empirique. Elle fait appel à la description et elle permet de savoir de quoi il s'agit et d'obtenir les éléments de base pour l'analyse. Au point de vue syntaxique, c'est le verbe de la phrase qui répond à cette question. Et on peut dans la plupart de cas nominaliser ce verbe et transformer l'énoncé auquel il appartient en titre thématique fort. Exemple : La fusée a explosé en vol : l'explosion de la fusée. De quoi parle-t-on? De quoi s'agit-il? De l'explosion de la fusée, bien sûr (Leclerc : 171). Cette question peut avoir pour variantes QU'EST-CE QUE, QU'EST-CE QUE C'EST. Elle permet aussi de trouver le concept générique. Selon les circonstances, on peut y répondre par une définition en compréhension (qui situe l'objet en indiquant dans quel ensemble il est compris) ou en extension (qui inventorie les composants de l'objet). Cette définition peut C'est aussi la question « leit-motiv » qui revient à chaque découverte d'un nouvel objet : « C'est quoi? », « Il faut faire quoi avec ça? » et qui répond à deux comportements exploratoires dont le premier consiste en une phase d'investigation où l'on se pose les questions «qu'est-ce que cet objet? », « quel est cet objet? », « de quoi s'agit-il? » et « quelles sont ses propriétés? », et le deuxième nommé « exploration diversive », où l'on s'intéresse à ce que l'objet peut faire ou ce que l'on peut faire avec l'objet (Piot-Ziegler 1988 : 94).

2) Les questions COMMENT et POURQUOI se posent au niveau analytique. COMMENT permet de savoir par quel moyen, de quelle manière ou de quelle façon un événement se produit. La réponse peut aller d'une action ponctuelle à toute une procédure comprenant plusieurs phases ou opérations (par exemple : la chaîne de production d'une bière ou d'une voiture). Cette question appelle généralement du concret, des réponses structurées, hiérarchisées avec des mises en ordre très souvent sous forme de mode d'emploi ou mode opératoire. On prend aussi en compte les moyens matériels utilisés (instruments, outils, ...), les moyens humains, les consignes, les modalités et les manières de la réalisation (Lorenzo 1992 : 119-120). POURQUOI permet de connaître les causes, les buts ou les objectifs de l'événement dont on parle ou de faire une recherche légitime des causes par une analyse de la situation. Ces deux questions demandent des réponses qui permettent d'aller au-delà de l'information reçue. Par exemple :

\footnotetext{
"Why did the balloon expand?", "How is heat or thermal energy conducted through a piece of iron?". Answering such questions involves observing, inferring, generalizing, and using prior knowledge [...] "Why" questions may not have a final answer; each successive answer may lead to another, more fundamental, question. For example, a student asks, "Why is grass green?" The teacher answers, "Because grass has chlorophyll," to which the students immediately asks, "Well, why is chlorophyll green?" This could go on indefinitely because each question leads back to more basic conceptional information. (Carin 1993 : 22).
} 
QUI : La question qui permet de connaître la personne, la chose ou le service qui est en cause. En science et en technique, cette question fait appel à la caractérisation de l'objet, donc à l'indication des attributs et des caractères intrinsèques ou extrinsèques.

OÙ : Permet de savoir le lieu où se trouve ou se produit quelque chose, ainsi que la destination de cette chose. C'est un complément circonstanciel qui répond à cette question. Cette question fait appel à des relations ontologiques ou à la compréhension spatiale (localisation) : village, région, site, grotte, à droite de ... On pourrait même, pour répondre à cette question, recourir à la typologie des lieux distingués selon la nature du terrain fournissant tel ou tel matériau de base, présentant tel climat.

QUAND : Permet de savoir à quel moment, pendant quelle durée et à quelle périodicité l'événement a lieu. Il faut tenir compte de la temporalité externe (le calendrier ordinaire, heure minute, etc.) mais aussi de la temporalité interne qui pour un processus de fabrication peut être l'échelle de chauffe ou juste la chronologie de la procédure logique des activités de fabrication ou d'intervention.

Il faut ajouter, bien évidemment une septième question tout aussi fondamentale que chacune des six que nous venons de présenter :

COMBIEN : Cette question permet de préciser la quantité ou la mesure de l'objet : le nombre, la quantité, le poids, la distance ...

Pour rendre l'investigation encore plus sérieuse et scientifique, nous pouvons affiner davantage notre sens critique et remettre en cause de manière systématique « toutes les données par l'application généralisée du POURQUOI à l'ensemble des réponses recueillies suite aux questions factuelles. [...] L'investigation est donc conduite dans un esprit de doute constructif, privilégiant la volonté de comprendre » (Bellenger 2000 : 35). Le tableau ci-après permet de prendre conscience de l'importance de cette démarche de questionnement :

QUOI et POURQUOI?

QUI et POURQUOI?

OÙ et POURQUOI?

QUAND et POURQUOI?

COMMENT et POURQUOI?

COMBIEN et POURQUOI? remet en question l'objet même de ce qui est à l'ordre du jour

remet en question les personnes, leur choix et leur qualification

remet en question le lieu, le contexte, le cadre, l'emplacement

remet en question l'ordre des opérations, leur découpage, leur périodicité, le moment, les temps, les durées

remet en question le procédé, le mode opératoire, la procédure, la méthode

remet en question dans les nombres, les opérations effectuées, les objets, les distances, les personnes.

(Bellenger $2000: 35$ ) 
Nous pensons que la méthode de questionnement systématique à partir des sept questions fondamentales que nous avons abordées peut développer l'esprit de curiosité des apprenants. La simplicité des questions contribuera, nous pensons, à démythifier les sciences et les techniques et à réduire le trac que beaucoup d'étudiants de traduction éprouvent face aux sujets scientifiques et techniques. Cette approche est tributaire des connaissances en terminologie et en science cognitive.

\section{RÉFÉRENCES}

BELlenger, L. et COUCHAERE, M.-J. (2000) : Les techniques de questionnement : savoir poser les bonnes questions, Issy-les-Moulineaux, ESF éditeur.

BertRAnd-Gastaldy, S. ET SAADANi, L. (2000) : « Cartes Conceptuelles et Thésaurus : Essai de Comparaison Entre Deux Modèles de Représentation Issus de Différentes Traditions Disciplinaires », in ACSI 2000 : ACSI 2000 : les dimensions d'une science de l'information globale, Association canadienne des sciences de l'information, Travaux du 28 e congrès annuel.

CABRÉ, M. T. (1998) : La terminologie : théorie, méthode et applications, traduit du catalan, adapté et mis à jour par Monique C. Cormier et John Humbley, Ottawa, Presses de l'Université d'Ottawa.

CARIN, A. A. (1993) : Teaching Modern Science, New York, Macmillan Publishing Company.

DUIT, R. et RHÖNECK, C. von (1998) : «Apprendre et comprendre les concepts clés de l'électricité », in

Tiberghien, A., JosSEM, L. E. et BAROJAS, J. : Des résultats de recherche en didactique de la physique à la formation des maîtres, Commission internationale sur l'enseignement de la physique (ICPE).

FABER, P. (1999) : "Conceptual analysis and knowledge acquisition in scientific translation", in Terminologie et traduction, 2.

HOWARD, R. W. (1987) : Concepts and schemata, an Introduction, London, Cassell Educational.

KeIL, F. C. (1989) : Concepts, kinds, and cognitives development, Cambridge (Mass.), The MIT Press.

L'Homme, M.-C. (2004) : La terminologie : principes et techniques, Montréal, Presses de l'Université de Montréal. Leclerc, J. (1999) : Le français scientifique : guide de rédaction et de vulgarisation, Brossard (Québec), Linguatech. LEMKE, J. L. (1990) : Talking science : language, learning and values, Norwood (New Jersey), Ablex Publishing Corporation.

LORENZO, G. Di (1992) : Questions de savoir : introduction à une méthode de construction autonome des savoirs, $2^{\mathrm{e}}$ édition, Paris, ESF éditeur.

Mintzes, J. J., Wandersee, J. H. And NovaK, J. D. (Eds) (2000) : Assessing Science Understanding: a Human Constructivist View, Toronto, Academic Press.

PIOT-ZIEGLER, C. (1988) : Maîtrise progressive des propriétés d'objets et formation de concepts dans une tâche de classification, Berne, Peter Lang. 